Research Paper

\title{
Development of a panel of serum $\lg G$ and $\lg A$ autoantibodies for early diagnosis of colon cancer
}

\author{
Meihong Chen ${ }^{1,2}$, Xiaoqing Lin1,3, Liangming Zhang1,3, Lili $\mathrm{Yu}^{1,3}$, Qingwei $\mathrm{Wu}^{1,3}$, Songgao Zhang1,3, \\ Fangqin Xue ${ }^{1,4}$, Yi Huang $1,3,5,6 \bowtie$ \\ 1. Provincial Clinical College, Fujian Medical University, Fuzhou 350001, China. \\ 2. Department of Clinical Laboratory, Fujian Provincial Hospital Jinshan Branch, Fuzhou 350001, China. \\ 3. Department of Clinical Laboratory, Fujian Provincial Hospital, Fuzhou 350001, China. \\ 4. Department of Gastrointestinal Surgery, Fujian Provincial Hospital, Fuzhou 350001, China. \\ 5. Center for Experimental Research in Clinical Medicine, Fujian Provincial Hospital, Fuzhou 350001, China. \\ 6. Key laboratory, Fujian Provincial Hospital, Fuzhou 350001, China. \\ $\square$ Corresponding author: Yi Huang, Department of Clinical Laboratory, Fujian Provincial Hospital, Fuzhou 350001, China. E-mail: hyi8070@126.com.
}

(1) The author(s). This is an open access article distributed under the terms of the Creative Commons Attribution License (https://creativecommons.org/licenses/by/4.0/). See http://ivyspring.com/terms for full terms and conditions.

Received: 2020.07.01; Accepted: 2020.09.14; Published: 2020.09.25

\begin{abstract}
Purpose: Our pilot study in a small cohort by ELISA showed that the levels and positive rates of serum IgG autoantibodies against p53, HRAS and NSGI, and IgA autoantibody against TIFIY in early colon cancer $(C C)$ group were significantly higher than that of colon benign lesion (CBL) group / healthy control (HC) group $(P<0.01)$, which suggested that four autoantibodies might be valuable for the diagnosis of patients with $C C$ at early stage. On the basis of pilot study, we intend to comprehensively elucidate the performance of four autoantibodies for the early diagnosis of CC in a large sample cohort, and explore the optimal panel of autoantibodies in the diagnosis of patients with $\mathrm{CC}$ at early stage.

Methods: Western blot was used to define the ELISA results of serum anti-p53, HRAS, NSG1-lgG and anti-TIFly-IgA. The performances of anti-p53, HRAS, NSG1-IgG and anti-TIFIY-IgA were evaluated by ELISA for the early diagnosis of CC with 601 serum samples of 157 patients with CC at early stage, 144 patients with CC at advanced stage, 130 patients with $\mathrm{CBL}$, and $170 \mathrm{HC}$, and then the performances of different combinations of four autoantibodies were analyzed for the development of an optimal panel for the early diagnosis of CC.

Results: The results of anti-p53, HRAS, NSG1-IgG and anti-TIFly-lgA in western blotting were consistent with that in ELISA. The levels and positive rates of anti-p53, HRAS, NSG1-IgG and anti-TIFly-IgA in early CC group were significantly higher than that in $\mathrm{CBL}$ group/HC group $(P<0.01)$, while had no significant difference from that in advanced $C C$ group $(P>0.05)$, of which anti-TIFl $Y$-lgA showed the highest area under the receiver operating characteristic curve (AUC) of 0.716 for the patients with CC at early stage, with $25.5 \%$ sensitivity and specificity at $96.7 \%$. Additionally, a panel of anti-p53, HRAS-IgG and anti-TIFly-lgA showed the highest AUC among all possible combinations of four autoantibodies, up to 0.737 , with $47.1 \%$ sensitivity at $92.0 \%$ specificity.

Conclusions: Serum IgG autoantibodies against p53, HRAS and NSG1, and IgA autoantibody against TIFly show the diagnostic value for the patients with CC at early stage, of which anti-TIFly-lgA is demonstrated to be a preferable biomarker, and an optimal panel comprised of anti-p53, HRAS-lgG and anti-TIFIY-IgA might contribute to the further improvement of early diagnosis for CC.
\end{abstract}

Key words: colon cancer, early diagnosis, autoantibody, combined detection

\section{Introduction}

Colon cancer (CC) is a common malignant tumor that seriously endangers human life and health. Globally, the incidence of colon cancer ranks $3^{\text {rd }}$ in males and $2^{\text {nd }}$ in females among all malignancies [1].
Early diagnosis and timely surgical radical resection are of great significance for the prognosis of CC patients. Previous studies have shown that 5-year survival in patients with early CC who undergo 
surgery could reach as high as $97 \%$ [2]. Unfortunately, due to the absence of apparent symptoms at the early stage, most of the patients present in the late stage of the disease, missing the best period for surgery.

As the traditional diagnostic method for colon cancer, fiber endoscopy and pathological tissue biopsies are applied in clinic for early CC detection, however, relatively complex operation, highly technical expertise requirement and uncomfortable invasive experience limit their application in the screening of asymptomatic populations. Therefore, improving the efficiency of early diagnosis of CC is becoming a clinical challenge [3]. Serological biomarkers have been highly recommended in the screening for early CC due to non-invasive, convenient and safe advantages; nevertheless, current conventional tumor antigen markers (Carcinoembryonic Antigen (CEA), CA19-9 and CA724, etc.) meet a different degree of detection sensitivity or specificity problems, which limit their clinical value for diagnosing CC [4]. Therefore, finding new serological markers for the early diagnosis of CC is essential.

Recent advances have demonstrated that autoantibodies against tumor-associated antigen (TAAs) exist in sera of $80 \%$ of cancer patients 3-5 years prior to the manifestation of clinical symptoms [5]. Hence, autoantibody detection is expected to dramatically aid in the diagnosis of malignancies at early stage [5-6]. Recently, using an approach based on HuProt array (v3.0; 20240 individual human proteins, provided by the High-throughput Biology Center of Johns Hopkins Medical College, USA), we successfully discovered and validated a total of eight IgG autoantibodies against p53, ETHE1, CTAG1A, C1QTNF1, TEX264, CLDN2, NSG1, and HRas, for lung cancer diagnosis at early stage [7]. In addition, in agreement with the advances of serum $\operatorname{IgA}$ autoantibodies for the diagnosis of some malignancies [8-9], we also performed the screening of serum IgA autoantibody biomarkers by HuProt array and found some IgA autoantibodies with good diagnostic value for early lung cancer, of which IgA antibody against TIF1 $\gamma$ was demonstrated the optimal diagnostic performance for the patients with lung cancer at early stage by validation of ELISA assay [10].

In view of the apparent advantages of serum autoantibodies for the early diagnosis of malignancies, whether above 8 IgG autoantibodies and anti-TIF1Y IgA could also meet the diagnosis of early $\mathrm{CC}$ is worth exploring. Interestingly, by detecting a small cohort of serum samples comprised of 30 patients with early CC, 30 patients with benign colon lesions (CBL) and 30 healthy controls (HC) using ELISA assay, we found that levels of IgG antibodies against p53, HRAS, NSG1 and IgA antibody against TIF1 $\gamma$, were significantly higher in patients with early CC than that in the CBL and HC group $(P<0.01)$. This suggested that these four autoantibodies might be valuable for the diagnosis of patients with early CC. Based on our pilot observation, we intended to comprehensively evaluate the diagnostic performances of four autoantibodies in a large cohort of serum samples and then determine an optimal panel for the early diagnosis of CC by comparing the values of all possible combinations of four autoantibodies in this study.

\section{Material and methods}

\section{Study subjects}

157 patients with colon cancer (CC) at early stage (TNM 0/I/II stage), 144 patients with CC at advanced stage (TNM III/IV stage), 130 patients with colon benign lesion (CBL), and 170 healthy controls (HC) were recruited from February 2016 to December 2018 at Fujian Provincial Hospital. All patients strictly met the diagnostic standards recognized by international or professional societies and none of the patients had accepted any treatment for the malignancy; the clinical and pathological data were shown in Table 1. $170 \mathrm{HC}$ participants received health examinations from the physical examination centre of Fujian Provincial Hospital and showed no evidence of disease, including malignancies, $\mathrm{CBL}$, etc., based on the colonoscopy. All CBL and CC patients were confirmed by pathological examination of tissue biopsies under the colonoscopy. Each subject was collected $5 \mathrm{ml}$ peripheral blood before the surgery and the serum was separated at $3000 \mathrm{rpm}$ for $5 \mathrm{~min}$ and stored at $-80^{\circ} \mathrm{C}$ before use. This study was approved by the Institutional Review Board of Fujian Provincial Hospital, and all participants provided written informed consent.

Table 1. Clinical data for the early $C C, C B L$ and $H C$ groups

\begin{tabular}{lllll}
\hline & CC $(\mathrm{n}=301)$ & CBL $(\mathrm{n}=130)$ & HC $(\mathrm{n}=170)$ & $P$ \\
\hline Age (years) & $62.1 \pm 7.2$ & $62.1 \pm 8.3$ & $61.0 \pm 7.7$ & 0.162 \\
Sex (\%) & & & & 0.551 \\
Male & $188(62.3)$ & $83(63.5)$ & $111(65.1)$ & \\
Female & $113(37.7)$ & $47(36.5)$ & $59(34.9)$ & \\
Staging (\%) & & & & \\
0 & $24(8.0)$ & & & \\
I & $36(12.0)$ & & \\
II & $97(32.2)$ & & \\
III & $107(35.5)$ & & \\
IV & $37(12.3)$ & & \\
CBL (\%) & & $56(43.1)$ & \\
Colonic adenoma & & $72(55.4)$ & \\
Colon hyperplastic polyps & & $2(1.5)$ & \\
Colon tuberculosis & & & \\
\hline
\end{tabular}




\section{Detection of four TAAs by immunohisto- chemistry (IHC) staining}

Formalin-fixed, paraffin-embedded 3-mm thick sections were deparaffinized and rehydrated, and then the sections were, respectively, incubated with primary antibodies against p53 (Abcam, UK) (1:100), HRAS (Abcam, UK) (1:100), NSG1 (Abcam, UK) (1:200), and TIF1Y (cell signaling technology, USA) (1:1000) for $18 \mathrm{~h}$ at $4{ }^{\circ} \mathrm{C}$ to carry out the standard IHC staining as described previously [10]. The outcome of IHC staining for 30 pairs of CC tissues and matched paracancerous tissues, randomly selected from 157 cases with CC at early stage, was manually evaluated and scored by two independent certified pathologists. The intensity of staining was graded as: $0=$ undetectable, $1+=$ weak staining, $2+=$ moderate staining and $3+=$ strong staining.

\section{Identification of four serum autoantibodies by western blot}

$200 \mathrm{ng}$ of denatured GST-tagged recombinant proteins, p53 (71 kDa), HRAS (46 kDa), NSG1 (48 $\mathrm{kDa})$, and TIF1Y (150kD) at (expressed by yeast and provided by CDI Laboratories, Inc., USA) and $50 \mathrm{ng}$ of denatured GST protein $(26 \mathrm{kDa})$ were subjected to vertical electrophoresis on $12 \%$ SDS-PAGE at $20 \mathrm{~mA}$, respectively. The separated proteins were electrotransferred onto the nitrocellulose membrane and incubated with sera (1:500 dilution for anti-p53, HRAS, NSG1-IgG detection, 1:100 dilution for antiTIF1y-IgA detection) or rabbit anti-GST antibody (Cwbiotech, China) $(1: 2000)$ at $4{ }^{\circ} \mathrm{C}$ overnight and the membranes were incubated with horseradish peroxidase-conjugated anti-human IgG (Cwbiotech, China) (1:5000), and anti-human IgA (Jackson Immuno, USA) (1:5000) to carry out the standard western blot assay as described previously [11]. The immunoreactivity was visualized by chemiluminescence imager (BioRad, USA).

\section{Detection of four serum autoantibodies using ELISA assay}

50 ng GST-tagged recombinant proteins, p53, HRAS, NSG1, and TIF1ץ were coated onto 96-well plates at $4{ }^{\circ} \mathrm{C}$ overnight, respectively, and then the nonspecific binding was blocked by $3 \%$ BSA at $37^{\circ} \mathrm{C}$ for $1 \mathrm{~h}$. The wells were incubated with serum samples (1:500 dilution for anti-p53, HRAS, NSG1-IgG detection, 1:100 dilution for anti-TIF1 $\gamma$-IgA detection) at $37{ }^{\circ} \mathrm{C}$ to carry out the standard ELISA assay as described previously [10], and immunoreactivity was measured by reading the A450. All assays were performed in duplicate, and the averaged OD value was calculated.

\section{Statistical analysis}

SPSS 22.0 statistical software was used to analyze the experimental data. For individual autoantibodies, cut-offs based on mean+2SD of HC group were used and $95 \%$ binomial confidence intervals were calculated for positivity percentage (sensitivity). For a panel of autoantibodies, CC designation was made if each autoantibody level exceeded the mean+2 SD of HC group. $X^{2}$ test was used for the comparison of rates between groups, and Mann-Whitney U-test was used for group comparisons. Graphpad Prism 5 and Medcalc v18.11 were used to draw charts and ROC curves, respectively. $P<0.05$ was considered a significant difference.

\section{Results}

\section{Expressions of four TAAs in early CC tissues}

The IHC staining results showed that four TAAs were highly expressed in early CC tusses, of which p53 and TIF1Y proteins were expressed in cell nucleus, HRAS protein was expressed in both cytomembrane and cytoplasm, and NSG1 protein was expressed in cytoplasm, while all four TAAs proteins were negative or weakly expressed in matched paracancerous tissues (Figure 1). The IHC positive rates for 2+-3+ staining of p53, HRAS, NSG1, and TIF1 $\gamma$ in early CC tissues were $60.0 \%, 56.7 \%, 60.0 \%$ and $66.7 \%$, respectively, and were significantly higher than $0.0 \%, 0.0 \%, 0.0 \%$ and $3.3 \%$ in paracancerous tissues $(P<0.01$, Table 2$)$.

Table 2. Comparison of positive rates of four TAAs expressions between early $\mathrm{CC}$ tissues and paracancerous tissues

\begin{tabular}{llllll}
\hline & $\mathrm{n}$ & \multicolumn{2}{l}{ Early CC tissues } & \multicolumn{2}{l}{ Paracancerous tissues } \\
\cline { 3 - 6 } & & $2+-3+$ & $0-1+$ & $2+-3+$ & $0-1+$ \\
\hline p53 & 30 & $60.0 \%(18 / 30)^{* *}$ & $40.0 \%(12 / 30)^{* *}$ & $0.0 \%(0 / 30)$ & $100 \%(30 / 30)$ \\
HRAS & 30 & $56.7 \%(17 / 30)^{* *}$ & $43.3 \%(13 / 30)^{* *}$ & $0.0 \%(0 / 30)$ & $100 \%(30 / 30)$ \\
NSG1 & 30 & $60.0 \%(18 / 30)^{* *}$ & $40.0 \%(12 / 30)^{* *}$ & $0.0 \%(0 / 30)$ & $100 \%(29 / 30)$ \\
TIF1 $\gamma$ & 30 & $66.7 \%(20 / 30)^{* *}$ & $33.3 \%(10 / 30)^{* *}$ & $3.3 \%(1 / 30)$ & $96.7 \%(29 / 30)$ \\
\hline \multicolumn{2}{l}{ Notes: **indicates compared with Paracancerous tissues, $P<0.01}$.
\end{tabular}

\section{Western blot validation of ELISA results}

GST-tagged recombinant proteins, p53, HRAS, NSG1, and TIF1 $\gamma$ were detected by western blot to validate the serum reactivity in ELISA, respectively. As shown in Figure 2, the serum of early CC patients with anti-p53, HRAS, NSG1-IgG (+), and anti-TIF1YIgA $(+)$ detected by ELISA only bound to the target protein, and not to GST-tagged protein; the serum of CBL patients and HC with anti-p53, HRAS, NSG1-IgG $(-)$, and anti-TIF1 $\gamma-\operatorname{IgA}(-)$ detected by ELISA did not bind to the target protein or GST- tagged protein. 

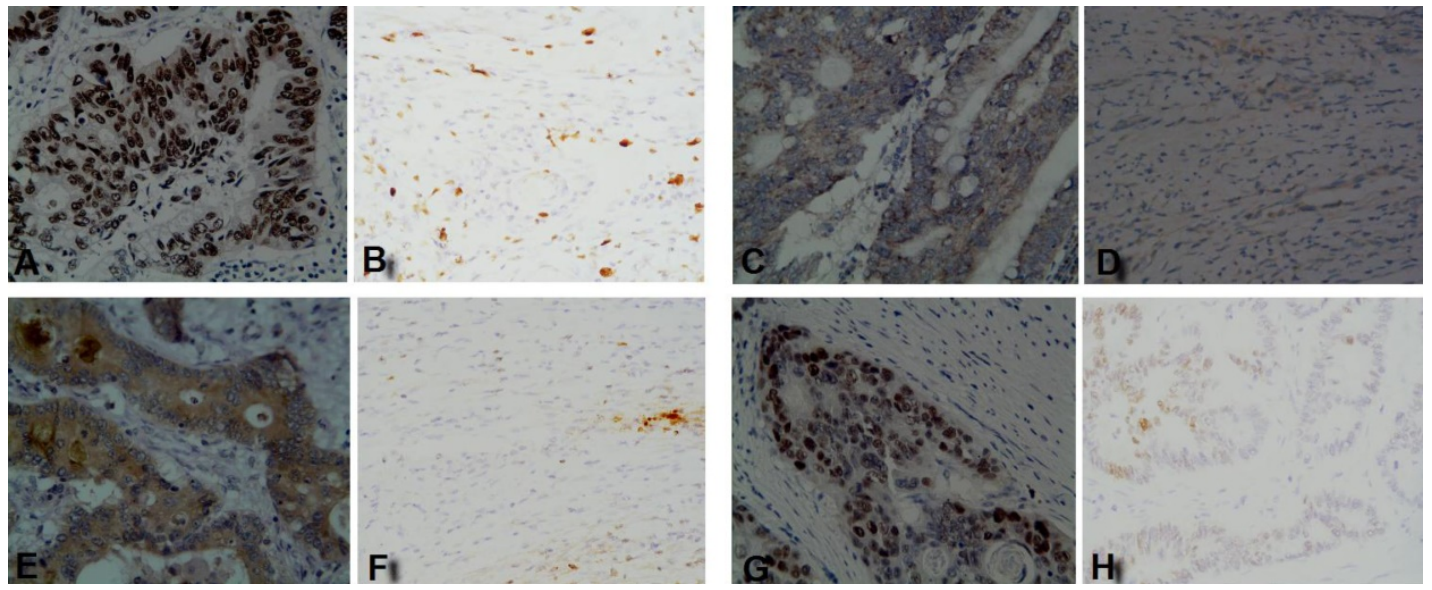

Figure 1. IHC staining results of four TAAs in early CC tissues and paracancerous tissues. (A, C, E, G) p53, HRAS, NSG1, and TIF1Y in early CC tissues, respectively; (B, D, $\mathbf{F}, \mathbf{H})$ p53, HRAS, NSG1, and TIF1Y in paracancerous tissues, respectively.
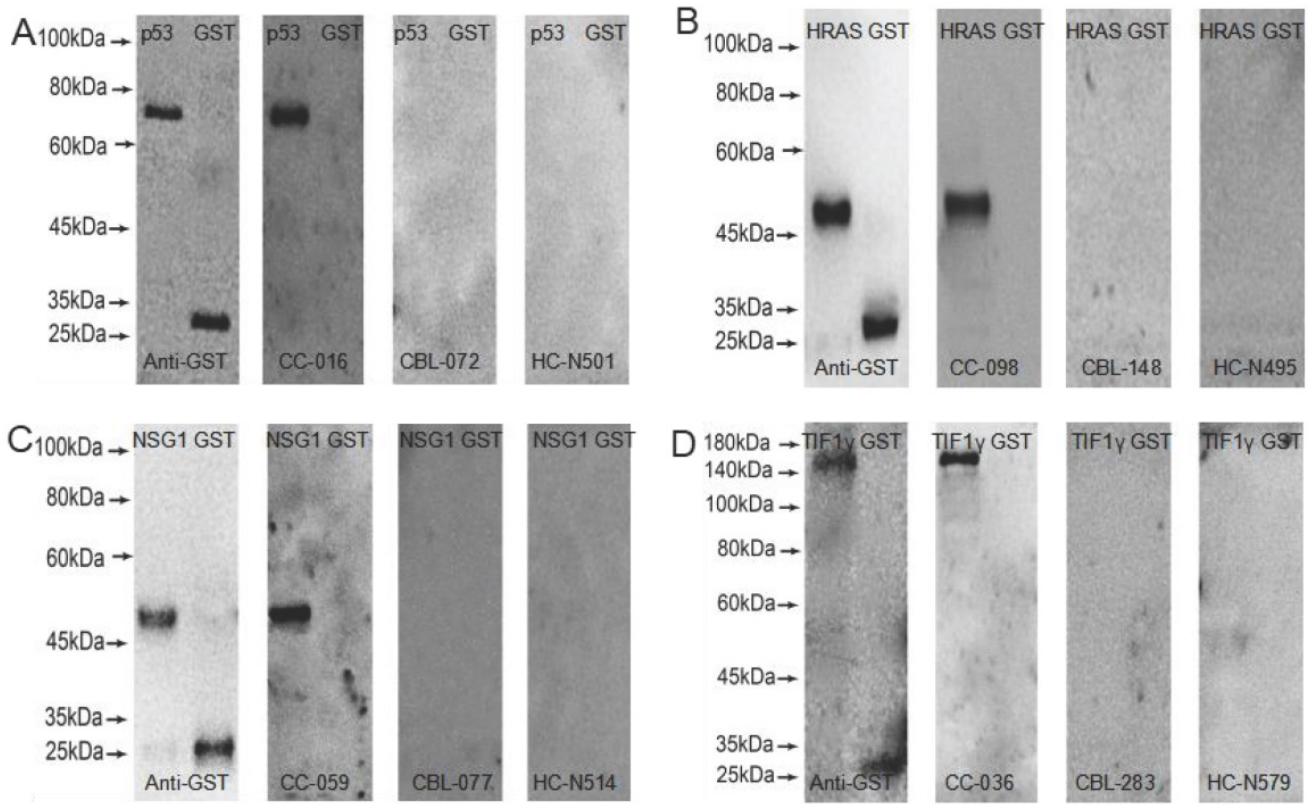

Figure 2. Western blot results of four serum autoantibodies. (A) p53-IgG; (B) HRAS-IgG; (C) NSG1-IgG; (D) TIFIY-IgA.

Table 3. Comparison of the positive rates of four serum autoantibodies in early CC, advanced CC, CBL and HC groups

\begin{tabular}{lllll}
\hline Marker & $\begin{array}{l}\text { Early CC } \\
(\mathrm{n}=157)\end{array}$ & $\begin{array}{l}\text { Advanced CC } \\
(\mathrm{n}=144)\end{array}$ & CBL $(\mathrm{n}=130)$ & HC $(\mathrm{n}=170)$ \\
\cline { 2 - 5 } & $\begin{array}{l}\text { Positive rate } \\
(\%)\end{array}$ & $\begin{array}{l}\text { Positive rate } \\
(\%)\end{array}$ & $\begin{array}{l}\text { Positive rate } \\
(\%)\end{array}$ & $\begin{array}{l}\text { Positive rate } \\
(\%)\end{array}$ \\
\hline p53-IgG & $23.6(37 / 157)^{* *}$ & $27.8(40 / 144)^{* *}$ & $4.6(6 / 130)$ & $2.4(4 / 170)$ \\
HRAS-IgG & $18.5(29 / 157)^{* *}$ & $20.8(30 / 144)^{* *}$ & $3.8(5 / 130)$ & $2.4(4 / 170)$ \\
NSG1-IgG & $20.4(32 / 157)^{* *}$ & $22.2(32 / 144)^{* *}$ & $6.2(8 / 130)$ & $3.5(6 / 170)$ \\
TIF1Y-IgA & $25.5(40 / 157)^{* *}$ & $25.7(37 / 144)^{* *}$ & $4.6(6 / 130)$ & $2.4(4 / 170)$ \\
\hline \multicolumn{5}{l}{ Notes: **indicates compared with CBL group/HC group, $P<0.01}$.
\end{tabular}

\section{Expressions of four serum autoantibodies}

The ELISA results showed that the levels and positive rates of anti- p53, HRAS, NSG1-IgG and anti-TIF1 $\gamma$-IgA in both early CC group and advanced CC group were significantly higher than that in CBL group and HC group $(P<0.01)$, while there was no significant difference in the levels and positive rates of four autoantibodies between early CC group and advanced CC group $(P>0.05)$ (Figure 3 , Table 3$)$. The AUC values of four autoantibodies for the patients with CC at early stage were $>0.600$, of which anti-TIF1Y-IgA showed the highest AUC of 0.716 (95\% CI $0.668-0.761$ ), with $25.5 \%$ sensitivity at $96.7 \%$ specificity (Figure 4, Table 4).

\section{Development of an optimal autoantibody panel}

Considering the comprehensive evaluation of the performances among all possible combinations of four autoantibodies, a panel of anti-p53, HRAS-IgG and anti-TIF1 $\gamma$-IgA showed the highest AUC for the patients with CC at early stage, up to $0.737(95 \% \mathrm{CI}$ $0.690-0.781$ ), with $47.1 \%$ sensitivity at $92.0 \%$ specificity (Table 4). 

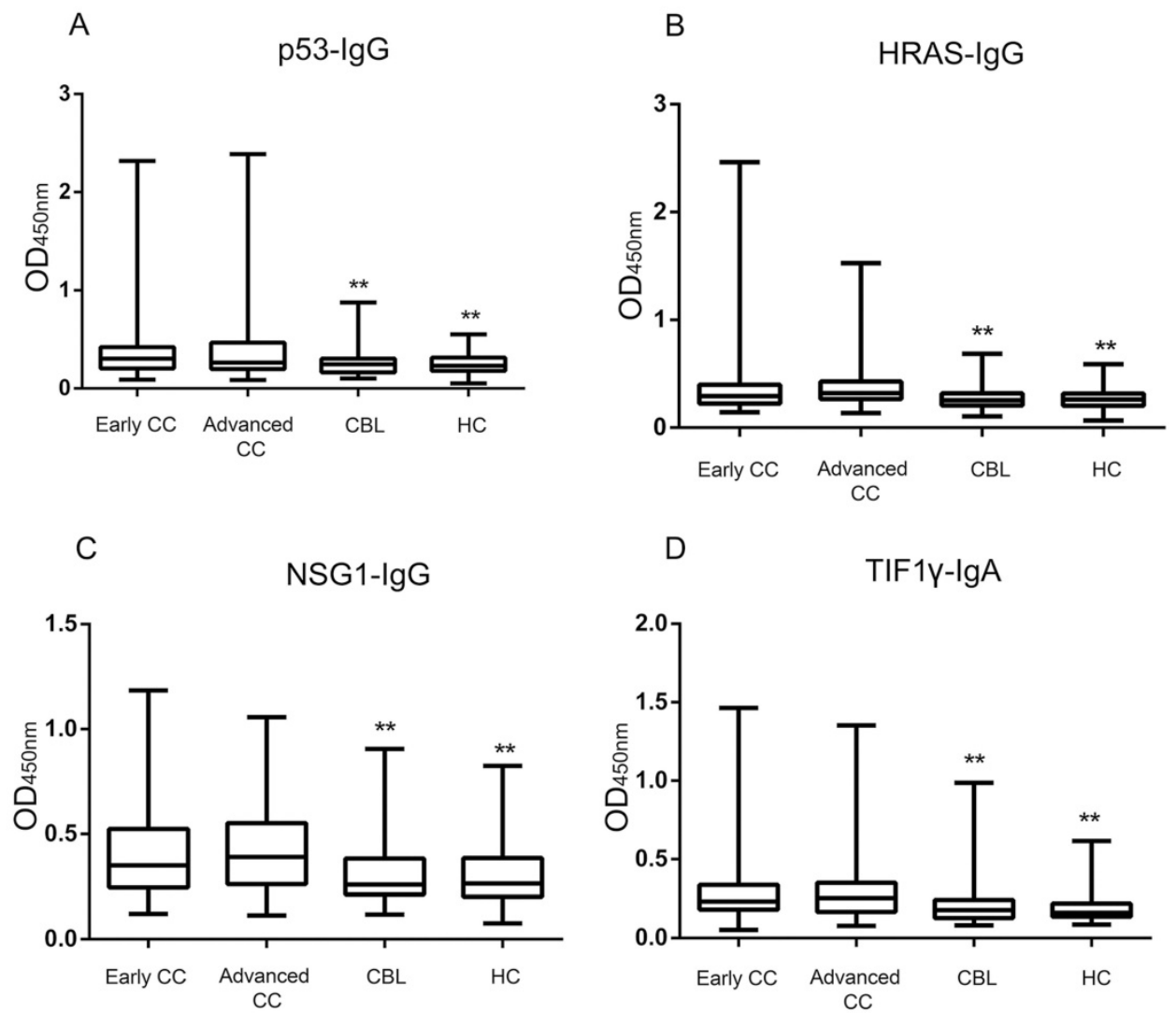

Figure 3. Comparison of four serum autoantibodies levels in early $C C$, advanced $C C, C B L$ and $H C$ groups. ${ }^{*}$ : Compared with the early/advanced $C C$ group, $P<0.01$.

Table 4. Comparison of the performances of four serum autoantibodies in diagnosing the patients with $\mathrm{CC}$ at early stage

\begin{tabular}{|c|c|c|c|c|c|c|}
\hline Marker & $\begin{array}{l}\text { Sensitivity } \\
(\%)\end{array}$ & $\begin{array}{l}\text { Specificity } \\
(\%)\end{array}$ & AUC & SE & $95 \% \mathrm{CI}$ & $P$ \\
\hline p53-IgG & 23.6 & 96.7 & 0.644 & 0.032 & $0.594-0.692$ & $<0.001$ \\
\hline HRAS-IgG & 18.5 & 97.0 & 0.631 & 0.031 & $0.580-0.679$ & $<0.001$ \\
\hline NSG1-IgG & 20.4 & 95.3 & 0.649 & 0.030 & $0.599-0.697$ & $<0.001$ \\
\hline TIF1 $\gamma-\operatorname{IgA}$ & 25.5 & 96.7 & 0.716 & 0.027 & $0.668-0.761$ & $<0.001$ \\
\hline p53, HRAS-IgG & 34.4 & 94.0 & 0.680 & 0.031 & $0.630-0.726$ & $<0.001$ \\
\hline p53, NSG1-IgG & 38.2 & 92.0 & 0.688 & 0.031 & $0.639-0.734$ & $<0.001$ \\
\hline p53-IgG +TIF1 $\gamma$-IgA & 40.8 & 93.7 & 0.728 & 0.029 & $0.680-0.772$ & $<0.001$ \\
\hline HRAS, NSG1-IgG & 30.6 & 93.0 & 0.664 & 0.031 & $0.614-0.711$ & $<0.001$ \\
\hline HRAS-IgG+TIF1 $\gamma$-IgA & 35.7 & 95.3 & 0.724 & 0.028 & $0.676-0.769$ & $<0.001$ \\
\hline NSG1-IgG+TIF1 $\gamma-I g A$ & 35.7 & 92.7 & 0.710 & 0.028 & $0.662-0.755$ & $<0.0001$ \\
\hline p53, HRAS, NSG1-IgG & 44.6 & 90.0 & 0.695 & 0.031 & $0.646-0.741$ & $<0.001$ \\
\hline $\begin{array}{l}\text { p53, } \\
\text { HRAS-IgG+TIF1 } \gamma-\operatorname{IgA}\end{array}$ & 47.1 & 92.0 & $0.737^{* *}$ & 0.029 & $0.690-0.781$ & $<0.001$ \\
\hline $\begin{array}{l}\text { p53, } \\
\text { NSG1-IgG+TIF1 } \gamma-\operatorname{IgA}\end{array}$ & 46.5 & 89.7 & 0.727 & 0.029 & $0.680-0.771$ & $<0.001$ \\
\hline $\begin{array}{l}\text { HRAS, } \\
\text { NSG1-IgG+TIF1 } \gamma-\operatorname{IgA}\end{array}$ & 42.0 & 91.3 & 0.722 & 0.029 & $0.675-0.767$ & $<0.001$ \\
\hline $\begin{array}{l}\text { p53, HRAS, } \\
\text { NSG1-IgG+TIF1 } \gamma \text {-IgA }\end{array}$ & 51.6 & 88.0 & 0.735 & 0.029 & $0.688-0.779$ & $<0.001$ \\
\hline
\end{tabular}

Notes: ** indicate the highest AUC among all possible combinations of four autoantibodies;

Abbreviations: AUC, area under the curve; $\mathrm{SE}$, standar error; $\mathrm{CI}$, confidence interval.

\section{Discussion}

An increasing number of evidences have represented that large amounts of autoantibodies could be released into sera due to the trigger of humoral immune responses for TAAs during early tumorigenesis. As compared with the TAAs in sera, serum autoantibodies against TAAs have demonstrated superior sensitivity for the early diagnosis of cancer in asymptomatic patients; additionally, TAAs-associated autoantibodies present high stability and persistence in sera, and are easy to detect [12]. These excellent characteristics make it possible for TAAs-associated autoantibodies to serve as promising serological markers for the diagnosis of early CC [6]. Especially mentioned, all TAAs related with four autoantibodies in this study are presented to be closely related with tumorigenesis. p53, a wellknown tumor suppressor gene, is reported to be often mutated and overexpressed in various cancers, including CC [13-15], and responsible for the regulation of cell processes including cell cycle, apoptosis, senescence, and DNA repair [16-17]. HRAS is a member of the ras oncogene family, whose mutations are involved in the occurrence of bladder, thyroid, salivary ductal, epithelial-myoepithelial, and kidney cancer [18-19]. NSG1, an endosomal protein expressed in neuronal cells, is reported to be a transcriptional target of p53 based on the observation that some non-neuronogenous cancer cells can express NSG1 in a p53-dependent manner under the effect of hydrogen peroxide, doxorubicin, UV, and $\gamma$-ray. Furthermore, NSG1 overexpression presents 
the obvious effects on cell proliferation inhibition and cell apoptosis induction [20]. TIF1 $\gamma$, also known as TRIM33, ECTO, PTC7, and RFG7, is a regulatory factor of the TGF- $\beta /$ Smad pathway by inhibiting Smad4 [21-22]. Recent existing studies have revealed that TIF1Y could play key role in the process of tumorigenesis by regulation of cell proliferation, differentiation, migration and invasion [23]. By IHC staining, all four TAAs in this study are demonstrated
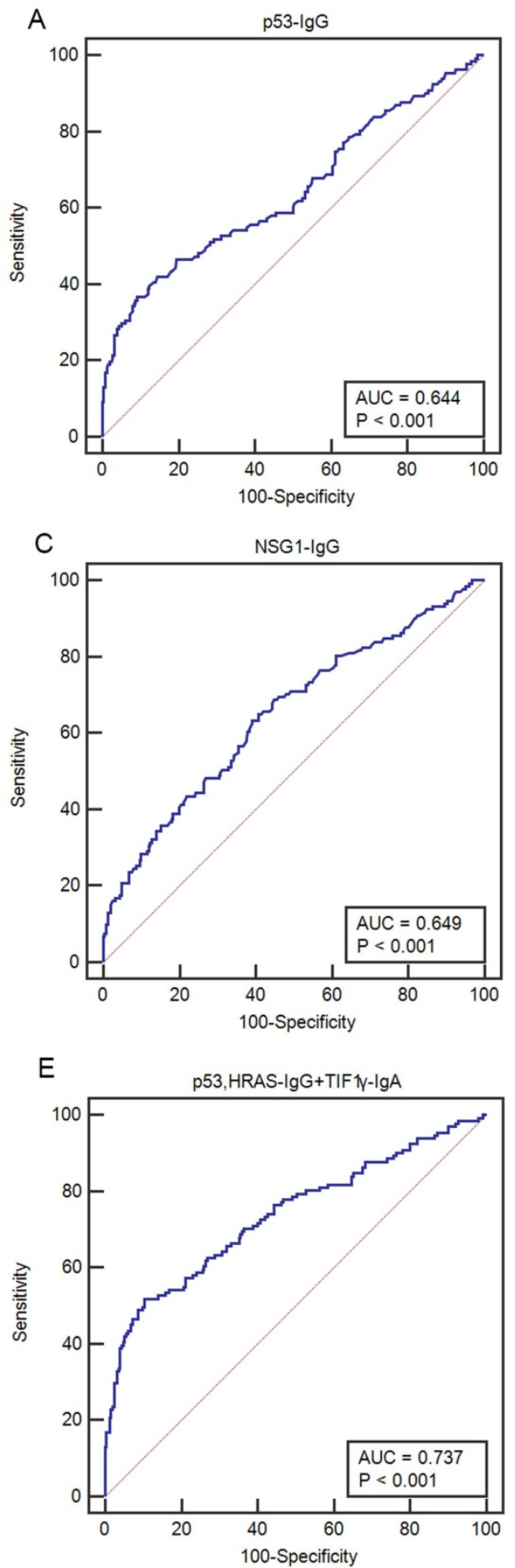

to be highly expressed in tumor tissues of early CC patients at the protein level, which might contribute to the aberrant release of autoantibodies in sera of patients with CC at early stage. Of four TAAsassociated autoantibodies, anti-p53-IgG is reliably detectable in sera of patients with CC [24]; however, the other three autoantibodies, anti-HRAS, NSG1-IgG and TIF1 $\gamma$-IgA, have not yet been described in the CC diagnosis.
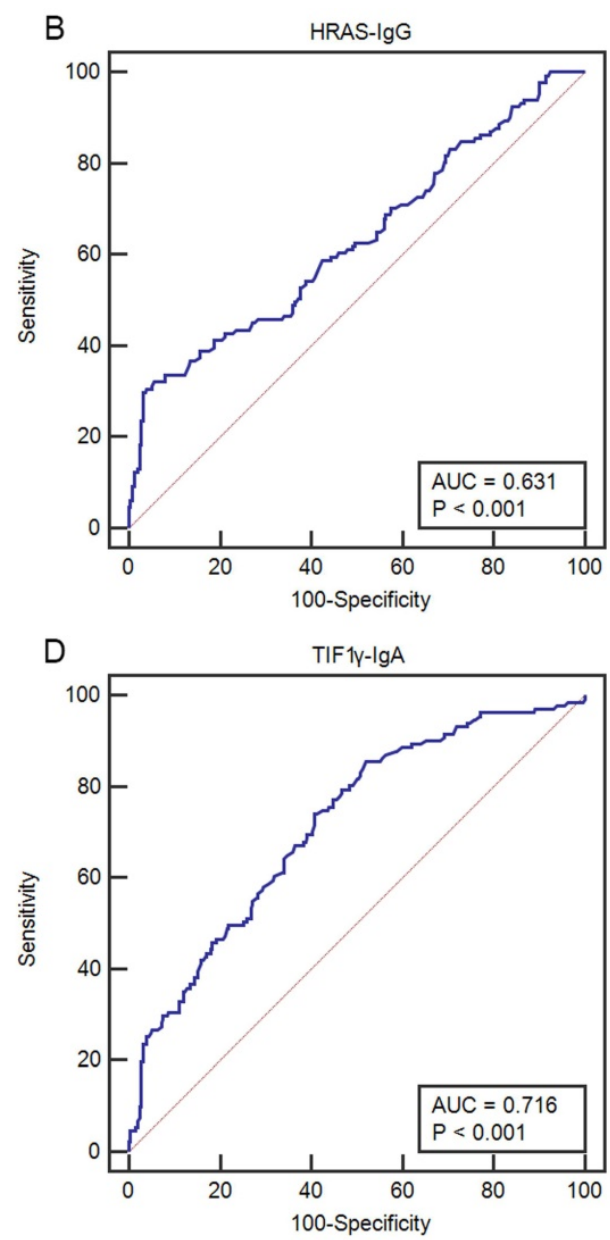

Figure 4. Comparison of ROC curves of four serum autoantibodies in patients with CC at early stage. (A-D) ROC curves for p53-lgG, HRAS-lgG, NSG1-lgG and TIF1Y-lgA, respectively; (E) ROC curve for an optimal panel of p53- IgG, HRAS-IgG and TIFIY-IgA. 
Considering our pilot observation that $\operatorname{IgG}$ autoantibodies against p53, HRAS and NSG1-IgG, and IgA autoantibody against IF1Y were highly expressed in sera of a small cohort of patients with early CC, we firstly proved the existence of a large amount of four autoantibodies in sera of early CC patients by the evidence that results of western blot were consistent with that of ELISA assay. And then, we performed the comprehensive evaluation of the diagnostic performances of four autoantibodies for patients with CC at early stage in a large cohort of up to 601 serum samples. Our results demonstrated the potentials of four autoantibodies for the early diagnosis of patients with CC. It was shown the levels and positive rates of four autoantibodies in early CC group were significantly higher than that in CBL and HC groups $(P<0.01)$, of which anti-TIF1 $\gamma$-IgA showed the best diagnostic performance of AUC of 0.716 , with $25.5 \%$ sensitivity at $96.7 \%$ specificity for the patients with early CC. In addition, there were no significant difference in the levels and positive rates of four autoantibodies between early CC group and advanced CC group $(P>0.05)$, indicating the fact that autoantibodies might be preferable indicators of early diagnosis other than illness monitoring for CC.

In view of the sensitivity of individual detection of serum anti-p53, HRAS, NSG1-IgG, and anti-TIF1 $\gamma$-IgA is relatively limited, we evaluated all possible combinations of these four autoantibodies. Encouragingly, the combination comprised of three autoantibodies, anti-p53, HRAS-IgG, and anti-TIF1YIgA, was determined to be an optimal panel for the diagnosis of early $\mathrm{CC}$, supported by its potential of improving AUC to 0.737 , with up to $47.1 \%$ sensitivity at $92.0 \%$ specificity for early CC. Finally, to the best of our knowledge, the diagnostic value of this panel of anti-p53, HRAS-IgG, and anti-TIF1 $\gamma$-IgA for early CC should be further proven by a multi-center research in the future and the further studies would be expected to carry out to excavate more valuable IgG and IgA autoantibodies to enhance the sensitivity and specificity for the diagnosis of early $\mathrm{CC}$, so as to further improve the early diagnostic efficiency and 5 -year survival rate of CC.

\section{Acknowledgments}

This study was supported in part by Scientific Joint Fund Of High Level Hospital Construction of Fujian Provincial Hospital (Grant No. 2017LHJJ04) to Y. Huang, Joint Fund Of Science And Technology Innovation of Fujian province (Grant No. 2017Y9069) to Y. Huang, Natural Science Foundation of Fujian Province (Grant No. 2019J01176) to Y. Huang, High-level Hospital Foster Grant of Fujian Provincial Hospital (Grant No. 2020HSJJ06) to Y. Huang.

\section{Competing Interests}

The authors have declared that no competing interest exists.

\section{References}

1. Bray F, Ferlay J, Soerjomataram I, et al. Global cancer statistics 2018: GLOBOCAN estimates of incidence and mortality worldwide for 36 cancers in 185 countries. CA Cancer J Clin. 2018; 68(6): 394-424.

2. Mahasneh A, Al-Shaheri F, Jamal E. Molecular biomarkers for an early diagnosis effective treatment and prognosis of colorectal cancer: Current updates. Exp Mol Pathol. 2017; 102(3): 475-83.

3. Menees SB, Kim HM, Elliott EE, et al. The impact of fair colonoscopy preparation on colonoscopy use and adenoma miss rates in patients undergoing outpatient colonoscopy. Gastrointest Endosc. 2013; 78(3): 510-6.

4. $\mathrm{Yu} \mathrm{JK}$, Yang MQ, Jiang TJ, et al. The optimal combination of serum tumor markers with bioinformatics in diagnosis of colorectalcarcinoma. Zhejiang Da Xue Xue Bao Yi Xue Ban. 2004; 33(5): 407-10.

5. Tan HT, Low J, Lim SG, et al. Serum autoantibodies as biomarkers for early cancer detection. FEBS J. 2009; 276(23): 6880-904.

6. Macdonald IK, Parsy-Kowalska CB, Chapman CJ. Autoantibodies: Opportunities for Early Cancer Detection. Trends Cancer. 2017; 3(3): 198-213.

7. Pan JB, Song G, Chen DY, et al. Identification of serological biomarkers for early diagnosis of lung cancer using a protein array-based approach. Mol Cell Proteomics. 2017; 16(12): 2069-78.

8. Erić-Nikolić A, Milovanović Z, Sánchez D, et al. Overexpression of calreticulin in malignant and benign breast tumors: relationship with humoral immunity. Oncology. 2012; 82(1): 48-55.

9. Yoneyama K, Shibata R, Igarashi A, et al. Proteomic identification of dihydrolipoamide dehydrogenase as a target of autoantibodies inpatients with endometrial cancer. Anticancer Res. 2014; 34(9): 5021-7.

10. Pan J, Yu L, Wu Q, et al. Integration of IgA and IgG Autoantigens Improves Performance of Biomarker Panels for Early Diagnosis of Lung Cancer. Mol Cell Proteomics. 2020; 19(3): 490-500.

11. Pan J, Zheng Q, Li Y, et al. Discovery and Validation of a Serologic Autoantibody Panel for Early Diagnosis of Esophageal Squamous Cell Carcinoma. Cancer Epidemiol Biomarkers Prev. 2019; 28(9): 1454-60.

12. Finn OJ. Immune response as a biomarker for cancer detection and a lot more. N Engl J Med. 2005; 353(12): 1288-90.

13. Ozaki T, Nakagawara A. Role of p53 in Cell Death and Human Cancers. Cancers (Basel). 2011; 3(1): 944-1013.

14. Munro AJ, Lain S, Lane DP. P53 abnormalities and outcomes in colorectal cancer: a systematic review. Br J Cancer. 2005; 92(3): 43-444.

15. Trivers GE, De Benedetti VM, Cawley HL, et al. Anti-p53 antibodies in sera from patients with chronic obstructive pulmonary disease can predate a diagnosis of cancer. Clin cancer Res. 1996; 2(10): 1767-75.

16. Shaw PH. The role of p53 in cell cycle regulation. Pathol Res Pract. 1996; 192(7): 669-75

17. Higashitsuji H, Masuda T, Liu Y, et al. Enhanced deactylation of p53 by the anti-apoptotic protein HSCO in association with listonedeacetylase 1. J Biol Chem. 2007; 282(18): 13716-25.

18. Prior IA, Lewis PD, Mattos C. A comprehensive servey of Ras mutations in cancer. Cancer Res. 2012; 72(10): 2457-67.

19. Andersson P, Kolaric A, Windahl T, et al. PIK3CA, HRAS and KRAS gene mutations in human penile cancer. J Urol. 2008; 179(5): 2030-4.

20. Ohnishi S, Futamura M, Kamino H, et al. Identification of NEEP21, encoding neuron-enriched endosomal protein of $21 \mathrm{kDa}$, as a transcriptional target of tumor suppressor p53. Int J Oncol. 2010; 37(5): 1133-41.

21. Dupont S, Zacchigna L, Cordenonsi M, et al. Germ-layer specification and control of cell growth by Ectodermin, a Smad4 ubiquitin ligase. Cell. 2005; 121(1): 87-99.

22. He W, Dorn DC, Erdjument-Bromage H, et al. Hematopoiesis controlled by distinct TIF1gamma and Smad4 branches of the TGFbeta pathway. Cell. 2006; 125(5): 929-41.

23. Chengpeng $\mathrm{Yu}$, Zeyang Ding, Huifang Liang, et al. The Roles of TIF1Y in Cancer. Front Oncol. 2019; 9: 979.

24. Tokunaga $R$, Sakamoto $Y$, Nakagawa $S$, et al. The utility of tumor marker combination, including serum P53 antibody, in colorectal cancer treatment. Surg Today. 2017; 47(5): 636-42. 